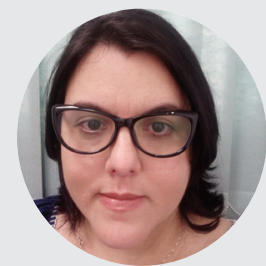

Susell Gómez González*

Coordinadora de

Estudios Generales

Instituto Tecnológico de

Santo Domingo (INTEC)

susell.gomez@intec.edu.do

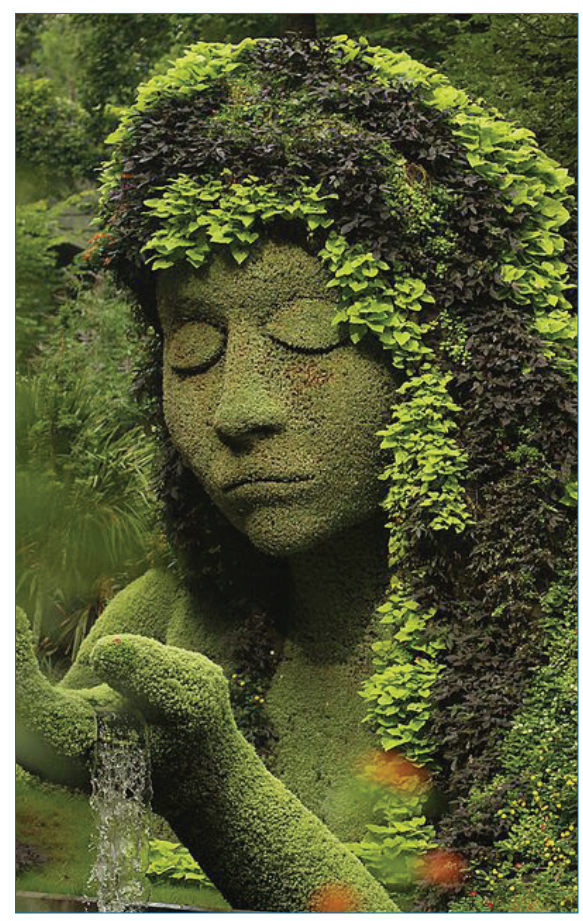

Esta edición no. 37 del Cuaderno de Pedagogía Universitaria, además de otros artículos propios de nuestro alcance, está dedicada a publicar parte de las ponencias ofrecidas en el XII Simposio Internacional de Estudios Generales (modalidad virtual), celebrado en junio 2021 en la Pontificia Universidad Católica Madre y Maestra (PUCMM), República Dominicana, y auspiciado por Red Internacional de Estudios Generales (RIDEG).

\title{
Editorial
}

\section{EXPERIENCIAS Y RETOS EN LOS ESTUDIOS GENERALES. BREVE ACERCAMIENTO DESDE LA TRANSDISCIPLINARIEDAD}

Los Estudios Generales revisten importancia e interés en el contexto universitario del continente. No obstante, las corrientes de análisis y pensamientos promovidos por esta mirada transdisciplinar con autonomía y voz propia encuentran, en ocasiones, resistencia y desarrollos disciplinares amparados en prácticas parceladas legitimadas.

La formación transdisciplinar de los Estudios Generales es un tema de gran interés, por lo que ha convocado una ola de artículos académicos y revisiones científicas. Rememorar estos esfuerzos incluye presentar autores tales como Manuel Maldonado, Waldemiro Vélez, David Álvarez, Enrique Mata Riera, María Elena Córdoba, Jorge Rodríguez Beruff, Ángel Quintero, Fidel Tubino, Pablo Quintanilla, Marino Grullon, Elvia Ojeda, entre otros, que, sin duda, constituyen la voz cantante para el posicionamiento del pensamiento latinoamericano, con énfasis en la formación general. Muchos de estos estudios ubican el tema de la transdisciplinariedad como una línea de proyección hacia la complejidad en los contextos socioculturales con una mirada crítica y exhaustiva sobre las prácticas docentes universitarias, fortaleciendo con mayor intención la concepción de una formación integral.

Uno de los principales esfuerzos para impulsar la educación general transdisciplinar ha sido la creación de la Red Internacional de Estudios Generales (RIDEG), cuya misión es aunar fuerzas para pasar de la inercia institucional a la práctica consciente. Sin embargo, aunque se reconozca la presencia oportuna de esta visión universitaria de alto alcance, la praxis indica, en algunos casos, brechas que, sin lugar a dudas, son oportunidades de mejoras. Por lo anterior, y pese a múltiples experiencias, es un imperativo ir más allá del discurso oficial de una o varias reformas curriculares para el ejercicio transdisciplinar. Esto corrobora una marcada existencia de redefiniciones curriculares que no siempre están amparadas bajo la mirada funcional

Lic. en Estudios Socioculturales. Coordinadora de Estudios Generales - Vicerrectoría Académica. Instituto Tecnológico de Santo Domingo (INTEC) Para contactar a la autora: Susell.gomez@intec.edu.do

ISSN (en línea): 1814-4152 / Sitio web: http://cuaderno.pucmm.edu.do

CóMO CITAR: Gómez, S. (2022). Editorial: Experiencias y retos en los Estudios Generales. Breve acercamiento desde la transdisciplinariedad.

Cuaderno de Pedagogía Universitaria, 19 (37), 5-7. 
y estratégica, coherente y oportuna al resto de las instancias universitarias: la emergencia de una coordinación de Estudios Generales, supeditada en algunos casos a áreas académicas varias, con la modalidad de agrupaciones disciplinares que retoman los paradigmas formativos del ciclo básico.

Resulta ambicioso definir experiencias de un proceso que se reconstruye contextualmente y es múltiple de acuerdo a los escenarios en los que se presenta. En un primer lugar, se constata el desarrollo de conceptos y aproximaciones presente en libros, compendios, artículos científicos, memorias de la realización del simposio y, por consiguiente, la legitimización de estas fórmulas, esto es, el punto de partida para hablar de experiencias. De todo esto deviene la conformación de la RIDEG como principal exponente de estas inquietudes, con recursos conceptuales y prácticos que hoy día son de obligada consulta en cualquier institución que decida proyectarse desde esta perspectiva. De igual forma, para asumir la relación formación general transdiciplinar hay todo un recurso teórico, ampliamente difundido, revisitado, comparado, publicado, que permite a los investigadores establecer los términos y replantearlos de acuerdo a los contextos reales en los que se desarrolla el proceso académico universitario, identificando su unívoca e inherente relación.

En apoyo a la búsqueda conceptual sobre el fin de los Estudios Generales se promulgan espacios de debate, se abren las dinámicas institucionales a nuevos retos y se logra proyectar la formación general en una idea que va más allá de completar un pensum, sistematizar contenido de la enseñanza precedente, o, simplemente, que el alumno adquiera sus créditos indispensables. Sino que, la misión de los Estudios Generales será “....proporcionar a nuestros alumnos una formación integral que articule lo humanístico, lo científico, lo artístico y lo tecnológico, de manera que ellos puedan estar en condiciones de transformarse a sí mismos, así como la sociedad y el mundo que los rodea" (Quintanilla, 2015, p. 4). El mismo autor anteriormente citado resume que el objetivo último de los Estudios Generales en la formación universitaria sería investigar temas que tienen que ver con el valor, analizando y cuestionando también lo valioso y significativo a través de los tiempos. (Quintanilla, 2015).
Pese al amplio campo de conceptualización sobre el deber ser de Estudios Generales constituido en documentos y conferencias, es posible que el componente transdiciplinar quede en el apartado de las formulaciones, sin embargo, la práctica y la gestión no son su mejor reflejo. Por lo cual, es necesario establecer dónde se está y a qué se aspira. De ahí surge la inquietud: ¿Qué experiencias y nuevos retos se presentan en la formación transdiciplinar de los Estudios Generales como base para la gestión y funcionamiento de esta área en la estructura universitaria?

En cuanto a las experiencias enunciadas en la pregunta, ellas son parte del gran y primer reto que inició con replantearse la formación general. Luego, el siguiente paso consistió en interiorizar la necesidad de la humanización del egresado, más allá de las artes y las letras y, por último, enfrentarse a la especialización reduccionista que permea el ciclo universitario. A estos pasos se sumó la definición de una gestión y su debida estructura, la definición de alcances y la adecuación de los reglamentos docentes a partir de nuevas funciones incorporadas.

Ahora, ante el sintagma 'nuevos retos' entendemos que no son nuevos, sino que se redimensionan trayendo cada circunstancia de acuerdo a las prácticas establecidas. Proceden de implementar las políticas curriculares a los contextos sociales, culturales, políticos, en los que se desarrollan nuestras universidades. No se encuentra una receta única en las gestiones consultadas, como tampoco una forma unívoca para hacer las cosas. Es por ello que, a pesar de logros innegables, se presentan interrogantes que ubican intrínsecamente una realidad que subyace en la praxis del día a día en la gestión y los procesos que se diluyen en el hacer por hacer.

En tal caso, se vuelven experiencias y logros innegables, pero, sigue latente la inquietud: ¿Son las áreas-facultades de nuestras universidades expresión de la concepción de formación general o siguen siendo espacios donde se fragmentan las asignaturas en escenarios disciplinares atendiendo a una estructura que no cambia aun con la transición curricular? De igual forma: ¿Qué tipo de capacitación y formación a los actores clave estamos ofreciendo hacia el ideal que aspiramos? Y, ¿nuestras prácticas y reglamentos son la expresión de la visión y 
objetivos que como universidades estamos trazando en pos de esa formación general integral? Por último, ¿Existe una visión real y oportuna de los Estudios Generales, o los cambios curriculares son la moda universitaria epidérmica que no cala en la funcionalidad operante diaria de las instancias que comparten esta responsabilidad?

Nuestras áreas-facultades, instancias y actores clave de la gestión podrían adolecer de una expresión sincera y consciente, no tanto de la concepción de formación general, sino de la aplicabilidad desde un pensamiento complejo, abierto, flexible, dispuesto al cambio y receptivo, por lo que es evidente encontrarnos con las formas y estrategias que forman parte de una cultura heredada y asumida en materia de gestión. En contraposición, los Estudios Generales deberían tener vida propia, deben ser un escenario ideal donde funcionen sus comunidades científicas y académicas, con espacio al debate y al diálogo. Además, debe ser un espacio curricular donde se efectúe el rediseño de programas, intercambio, vinculación y relación con la vida universitaria y que mantenga una relación directa con el contexto de la nación y sus políticas. No podemos cambiar el currículo sin la responsabilidad de la formación y cambios que devendrán con este.

Este ejercicio de reflexión incluye a todos y, por tanto, implica una coherencia entre los planes de estudios, las asignaturas y la gestión universitaria y no pretende des-construir las experiencias, sino establecer una reflexión sincera desde la praxis, lo que conlleva una revisión interna. No podemos obviar que hay un claro entendimiento en muchas de nuestras universidades de los Estudios Generales, pero se debe ser más explícito en los procesos. Los cambios curriculares no deben ser epidérmicos, sino que calen profundamente en la funcionalidad operante diaria de las instancias que comparten esta responsabilidad. Por lo que, es importante impulsar reformas que se socialicen y se retroalimenten de sus contextos y con sus actores clave. En consecuencia, el docente de Estudios Generales ha de sentir que pertenece, pero para ello debe primero la institución responsabilizarse de adecuar las vías para que esto llegue a feliz término y procurar la formación permanente de este grupo sensipensante.
Es de todos sabido que las instituciones en sus modos de operar han de ser el reflejo de la tarea de formalizar la enseñanza general como un componente innegable y así debe ser explicado, abordado y desarrollado desde y para los actores clave (alumnos, profesores, coordinadores, decanos, personal que interviene en el proceso de selección, registro, vida universitaria, etc.). Pero, además de colocar este ciclo en su estructura formal, deben permanecer reflexionando: ¿Cómo lograr la transdiciplinariedad en la gestión de Estudios Generales? ¿Es posible? Definitivamente que sí, sin embargo, lograrlo implica que todos los involucrados asumamos de forma consciente la misma y le demos paso, perdamos el miedo a dejar de ser protagonistas y pasemos a ser mediadores. Para que se logre, debemos permitir que las necesidades socioculturales de nuestros alumnos signen el camino. Hagámoslo a la inversa, estudiemos el contexto y luego proyectemos la gestión de programas que se parezcan más a nuestro tiempo y que se acerquen a ese ideal humanístico, con una flexibilidad y alcance que revista esa bienvenida que se espera de los Estudios Generales en la universidad. En definitiva, "las buenas universidades se distinguen, no solo por sus aportaciones a la investigación, sino por la formación humanística que proveen a sus estudiantes". (Rodríguez, 2010, p. 10).

\section{Referencias bibliográficas}

Quintanilla, P. (2015). Conocimiento, competencias y habilidades. Pontificia Universidad del Perú. Sobre formación permanente y docencia en los Estudios Generales. Documentos 22. Instituto Tecnológico de Santo Domingo. ISSN: 03789656X

Rodríguez, J. (2010). Los estudios generales en la Universidad de Puerto Rico: notas sobre un cambio curricular. Cuaderno de Pedagogía Universitaria. Año 7. N. 14, julio - diciembre. Santiago, República Dominicanal pp.6-10. https://www.pucmm.edu.do/publicaciones/ Documents/cuaderno-pedagogia/CuadernodePedagogiaNo14.pdf 